\title{
Cross-Modality Assessment and Planning for Pulmonary Trunk Treatment Using CT and MRI Imaging
}

\author{
Dime Vitanovski ${ }^{1,2}$, Alexey Tsymbal ${ }^{1}$, Razvan Ioan Ionasec ${ }^{1}$, \\ Bogdan Georgescu ${ }^{1}$, Martin Huber ${ }^{1}$, Andrew Taylor ${ }^{3}$, Silvia Schievano ${ }^{3}$, \\ Shaohua Kevin Zhou ${ }^{1}$, Joachim Hornegger ${ }^{2}$, and Dorin Comaniciu ${ }^{1}$ \\ 1 Integrated Data Systems, Siemens Corporate Research, Princeton, USA \\ 2 Pattern Recognition Lab, Friedrich-Alexander-University Erlangen-Nuremberg, \\ Germany \\ ${ }^{3}$ Great Ormond Street Hospital for Children, London, England
}

\begin{abstract}
Congenital heart defect is the primary cause of death in newborns, due to typically complex malformation of the cardiac system. The pulmonary valve and trunk are often affected and require complex clinical management and in most cases surgical or interventional treatment. While minimal invasive methods are emerging, non-invasive imagingbased assessment tools become crucial components in the clinical setting. For advanced evaluation and therapy planning purposes, cardiac Computed Tomography (CT) and cardiac Magnetic Resonance Imaging (cMRI) are important non-invasive investigation techniques with complementary properties. Although, characterized by high temporal resolution, cMRI does not cover the full motion of the pulmonary trunk. The sparse cMRI data acquired in this context include only one $3 \mathrm{D}$ scan of the heart in the end-diastolic phase and two 2D planes (long and short axes) over the whole cardiac cycle. In this paper we present a cross-modality framework for the evaluation of the pulmonary trunk, which combines the advantages of both, cardiac CT and cMRI. A patient-specific model is estimated from both modalities using hierarchical learning-based techniques. The pulmonary trunk model is exploited within a novel dynamic regression-based reconstruction to infer the incomplete cMRI temporal information. Extensive experiments performed on 72 cardiac CT and $74 \mathrm{cMRI}$ sequences demonstrated the average speed of 110 seconds and accuracy of $1.4 \mathrm{~mm}$ for the proposed approach. To the best of our knowledge this is the first dynamic model of the pulmonary trunk and right ventricle outflow track estimated from sparse $4 \mathrm{D}$ cMRI data.
\end{abstract}

\section{Introduction}

Congenital Heart Defect (CHD) is the primary cause of death in newborns characterized by complex malformations of the heart and great vessels. Often, the right side of the heart is affected and especially the pulmonary trunk, as in Tertalogy of Fallot (TOF) and pulmonary artesia or stenosis. The clinical management 
of such conditions is confronted with complex treatment decisions, which include pulmonary valve procedures in the majority of the cases.

Percutaneous interventions for pulmonary valve replacement are emerging as feasible treatment alternatives to the classical cardiac surgery with important benefits: less invasive, reduced risks associated with cardiopulmonary bypass, bleeding, infections and reduced expenses for postoperative intensive care 11. Nevertheless, comprehensive investigation, based on non-invasive imagine techniques, is still mandatory for clinical decision making and treatment success.

For therapy planning purposes, the pulmonary trunk is increasingly imaged using either cardiac Computer Tomography (CT) or cardiac Magnetic Resonance Imaging(cMRI) 2]. While CT has a high spatial resolution, fast acquisition times without anesthesia, it has the disadvantages of poor temporal resolution and ionizing radiation. Contrary, cMRI has high temporal resolution without Xray radiation, but long acquisition times and usually does not cover the full $4 \mathrm{D}$ information. The regular protocol, so called sparse 4D cMRI, involves an end-diastolic (ED) 3D heart image and two orthogonal cine projections $2 \mathrm{D}+\mathrm{t}$, short axis (SA) and long axis (LA). LA passes through the main pulmonary artery and the descending aorta, while SA is aligned with pulmonary valve, perpendicular to the LA (see Fig. 1). Ideally, clinicians would be provided with a accurate morphological and functional quantification of the pulmonary trunk, independent of the employed imaging technique.

In this paper we present a cross-modality framework for the evaluation of the pulmonary trunk, which combines the advantages of both, cardiac CT and cMRI, non-invasive imaging techniques. A physiological model, which captures complex morphological, dynamic and pathologic variations of the pulmonary trunk is presented in Sec. 2. In Sec. 4 the patient-specific model parameters are estimated from both modalities within a hierarchical learning-based framework, which involves three-stages: landmark detection, center line detection and dynamics estimation. A novel dynamic regression-based reconstruction is proposed in Sec. 3 to infer the incomplete temporal information characteristic to the sparse cMRI protocols is presented.

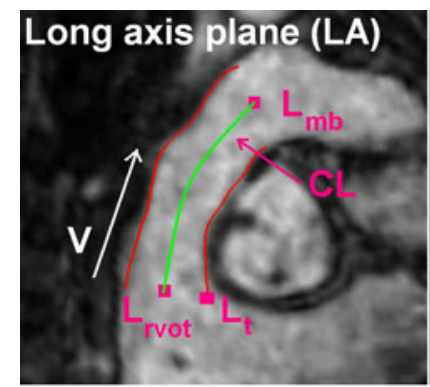

a)

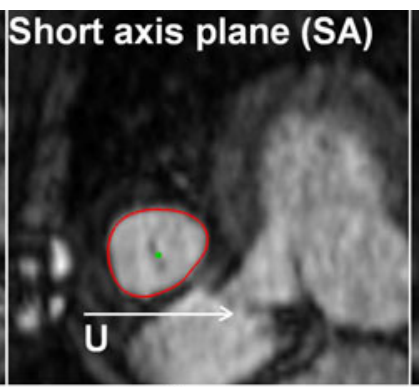

b)

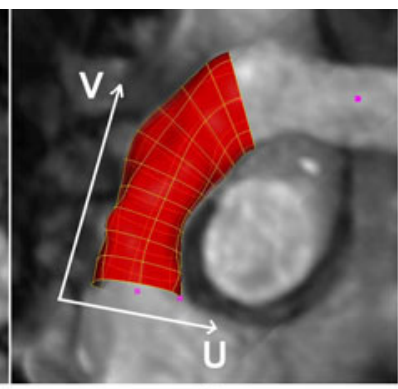

c)

Fig. 1. 3D cMRI scan of the whole heart in the ED phase (a). 2D long axis (LA) plane (b) and short axis (SA) plane (c) of the pulmonary artery over the cardiac cycle. 


\section{Physiological Pulmonary Trunk Modelling}

In this section we introduce our physiological model of the RVOT and pulmonary trunk, which represents both morphological and dynamic variations. Similar to [3], the anatomical complexity is reduced by employing a coarse to fine parameterization which includes: anatomical landmarks, pulmonary artery center line and the full surface model of the pulmonary trunk. As illustrated in Fig. 1(a), the considered anatomical landmarks include, Trigone $\left(L_{t}\right)$, RVOT $\left(L_{\text {rvot }}\right)$ and Main-Bifucation $\left(L_{m b}\right)$, each represented in the Euclidean 3D space, $L_{x} \in R^{3}$. The centre line $C L$ passes through the pulmonary artery center and is parameterized by 12 points, $C L=C L_{0} \ldots C L_{11}$. The surface model $S$ is represented by a structured grid, spanned along two anatomical directions, $u$-circumferential and $v$-longitudinal, using $50 \times 40$ vertices (see Fig. 1(c)). Point correspondence in time and across patients is enforced by intrinsic resampling of $S$, using a set of anatomical-driven cutting-planes, described by center line points $C L_{x}$ and corresponding tangential directions. Given the different modalities supported and characteristic imaging protocols, we differentiate among two dynamic extensions of the proposed physiological model. The definition of a full $4 \mathrm{D}$ model, which can be directly estimated from $4 \mathrm{D}$ cardiac CT data, is rather straightforward and realized by plain concatenating a time variable $t$ :

$$
\text { Model }_{\text {full } 4 D}=\left\{L_{t}, L_{\text {rvot }}, L_{m b}, C L, S\right\}_{t}
$$

However, given the sparse 4D acquisition, common to cMRI, the extension to a temporal model includes two additional representations: $L A$ and $S A$. $L A$ which describes the contour of $S$ intersected with the plane with the origin in $L_{r v o t}$ and the normal obtained from the cross-product between $L_{\text {trigone }}$ and $L_{\text {rvot }}$, and the center line tangent at $C L_{0}$ represents a specific $2 \mathrm{D}+$ time long axis acquisition (Fig. 1(a)). $S A$ describes the contour of $S$ intersected with the plane center in $C L_{\text {middle }}$ and the corresponding tangent as normal (Fig. 1(b)). Hence, the sparse dynamic model is parameterized as follows:

$$
\text { Model }_{\text {sparse } 4 D}=\left\{L_{t}, L_{\text {rvot }}, L_{m b}, C L, S\right\}_{E D}+\{L A, S A\}_{t}
$$

\section{Dynamic Regression Based 4D Model Reconstruction}

As describe above, the dynamic information in case of $4 \mathrm{D}$ cMRI is incomplete but rather available only in two orthogonal projections, as opposed to 4D cardiac $\mathrm{CT}$, which provides full dynamics over the cardiac cycle.

In regression a solution to the following optimization problem is normally sought [4:

$$
\hat{\mathcal{R}}(\mathbf{x})=\operatorname{argmin}_{\mathcal{R} \in \Im} \sum_{n=1}^{N} L\left(y\left(\mathbf{x}_{n}\right), \mathcal{R}\left(\mathbf{x}_{n}\right)\right) / N
$$

where $\Im$ is the set of possible regression functions, $L(\circ, \circ)$ is a loss function that penalizes the deviation of the regressor output $\mathcal{R}\left(\mathbf{x}_{n}\right)$ from the true output, and 
$N$ is the number of available training examples. In our case the reconstruction task is defined as a regression problem between the full dynamic model of the pulmonary trunk extracted from 4D CT data and the sparse one extracted from the sparse cMRI data:

$$
Y\left(\text { Model }_{\text {full } 4 D}\right)=\hat{\mathcal{R}}\left(X\left(\text { Model }_{\text {sparse } 4 D}\right)\right)+\epsilon
$$

In our regression problem we focus on shape information and completely neglect volume data. As descriptors both for the input $X\left(M_{\text {odel }}\right.$ sparse $\left.4 D\right)$ and output elements $Y\left(\right.$ Model $\left._{\text {fulluD }}\right)$ of the models we choose coordinates of mesh vertices normalized with the generalized procrustes analysis. This representation has a uniform representation of the input and the output data. The training set $T$ used to generate the regression model thus includes feature vectors $T_{i}$ as follows:

$$
T_{i}=<\left(S_{i}^{E D}, L A_{i}^{t}, S A_{i}^{t}\right)_{M R I},\left(S_{i}^{t}\right)_{C T}>,
$$

where $t$ is a time step within the cardiac cycle, $S_{i}^{E D}$ is a set of 3D coordinates representing each point of the end-diastolic model (2000 3D points), $L A_{i}^{t}$ and $S A_{i}^{t}$ are point sets ( 80 and $503 \mathrm{D}$ points respectively) representing the model curves extracted from the cMRI's long axis stack and short axis stack respectively, for the current time step $t$, and $\left(S_{t}^{i}\right)_{C T}$ are the corresponding point coordinates for the point set to be reconstructed (238 3D points). Due to the dense representation of our model (2000 3D points) we reconstruct only the most significant 238 $3 \mathrm{D}$ points from the associated $\mathrm{CT}$ model. The rest of the points are interpolated and projected onto the PCA shape space from which the complete model is then obtained.

The formulated regression problem is solved by learning the regression function $\mathcal{R}$ with two different methods: boosting-based additive regression [5] and random forest [ 6 . Two main reasons motivate our choice. First, these techniques were shown to be robust to high-dimensional data with many irrelevant, redundant and noisy features, without the need for additional data pre-processing and feature selection. This was shown both for classification [7,,$\underline{8}$ and regression [4] tasks. Second, both boosting-based and random forest-based models are relatively fast to train and to evaluate comparing for example with Support Vector Regression. In the spirit of [7,,4], we use simple $1 D$ linear regression as the base learner for boosting-based regression. Each weak learner is a simple linear regressor of the form:

$$
y=\beta_{0} x+\beta_{1}
$$

where $x$ is the selected scalar input coordinate and $y$ is a scalar output coordinate. Using more sophisticated weak learners such as CART decision trees and multiple linear regression with greedy forward feature inclusion, has proven to always result in a worse or no better performance while the resulting model gets significantly more complicated. Using simple $1 \mathrm{D}$ binary decision stumps as in 4 . has also proven to lead to suboptimal accuracy; the reason for this is perhaps the nature of the data, as it is rather impossible to generate as many candidate decision stumps with the coordinate - based features as it is possible with 
the Haar-like features. For each boosting-based model, we generate 200 weak learners. The accuracy plateaus with this number of component models, and the further accuracy increase is always insignificant with this data.

For random forests, we always generate 25 component trees. The accuracy usually remains same or even decreases with the addition of more trees to the model. The minimum leaf size is set to 1 ; the trees are thus generated to the full with no pruning. The number of features considered at each node is set to the value recommended by Breiman [6, which is one third of the total number of features for regression. Using other parameter settings was shown to lead to worse or no better accuracy in our preliminary experiments.

In boosting-based regression the output function is assumed to take a linear form as follows [4]:

$$
\hat{\mathcal{R}}(\mathbf{x})=\sum_{t=1}^{T} \alpha_{t} h_{t}(x) \in H
$$

where $h_{t}(x)$ is a base (weak) learner and $T$ is the number of boosting iterations. Having a linear base learner (simple linear regression), a linear final solution is thus also found. In contrast to this, random forests seek for a non-linear function approximation, recursively splitting the feature space in the nodes of component decision trees.

In contrast to [4, we use naive decoupling of the regression problem into a number of single output problems. While multi-output regression solutions do exist both for boosting 4], for our task multi-output optimization was not shown to lead to error decrease and time savings were rather insignificant.

\section{Estimating Patient-Specific Model Parameters}

The patient-specific model parameters described in Section 2 are estimated from cardiac acquisition using a learning-based approach. Detectors are learned separately for both modalities, CT and cMRI, and applied to estimate model parameters in a hierarchical three-stage approach: Anatomical Landmarks Estimation, Center Line Estimation and Full Surface Model Estimation.

Anatomical Landmarks Estimation. By defining the localization as a classification problem, the anatomical landmarks, $L_{t}, L_{r v o t}, L_{m b}$, are estimated within the Marginal Space Learning (MSL) framework 9. Separate detectors $D_{t}^{L}, D_{r v o t}^{L}, D_{m b}^{L}$, are learned using the Probabilistic Boosting Tree (PBT) [8] in combination with Haar-like feature from a training dataset annotated by experts.

$$
p\left(L_{x} \mid x, y, z\right)=D_{x}^{L}(x, y, z),(x, y, z) \in \sigma^{x}
$$

The trained detectors $D_{x}^{L}$ models the target posteriori distribution $p\left(L_{x} \mid x, y, z\right)$ for a specific search space $\sigma^{x}$ formed by the training set. MSL is applied to conduct guided search in the parameter space using the learned detectors and obtained the location of the anatomical landmarks. Note that in case of $4 \mathrm{D}$ cardiac CT anatomical landmark are detected in each volume to obtain the dynamic parameters $\left\{L_{t}, L_{\text {rvot }}, L_{m b}\right\}_{t}$, while in sparse cMRI only a static detection in the end-diastolic volume is performed: $\left\{L_{t}, L_{r v o t}, L_{m b}\right\}_{E D}$, 
Center Line Estimation. $C L$ passes through the centre of the pulmonary trunk and is initialized by the previously detected landmarks $L_{t}$ and $L_{r v o t}$. A robust detector $D^{C L}$ is learned using the same MSL framework to detect circular structures, parameterized by center line points $C L_{x}$, corresponding tangent and having fixed radius $r=20 \mathrm{~mm}$ obtained from the average value in the training set. An incremental approach is used to search circles on a series of successively updating planes. Please note, that for the Anatomical Landmarks Estimation, a temporal center-line model $C L_{t}$ is detected in $\mathrm{CT}$ and a static $C L$ in cMRI.

Full Surface Model Estimation. The full model of the pulmonary trunk $S$ is initialized in the end-diastolic frame using the estimated landmarks and centerlines, using a piecewise affine transformation along the center line 3. Robust boundary detectors $D^{s}$, trained using the PBT and steerable feature 9 are applied to locally refine the surface by moving it along normal directions towards the position with the highest boundary probability. To obtain spatially smooth delineation, the final results are obtained by projecting $S$ to a previously learned shape space model.

In case of $\mathrm{CT}$, the above described algorithm is applied in each time step to obtain the full temporal model $\left\{L_{t}, L_{r v o t}, L_{m b}, C L, S\right\}_{t}$. In case of MR, the estimated surface in the end-diastolic frame $S_{E D}$ is used to initialize the contours $L A$ and $S A$. These are refined using a trained $D^{c}$ contour detector as described above. A full dynamic $4 \mathrm{D}$ model is then estimated by using a learned regression model (see Eq. 4) to predict the missing temporal information.

\section{Results}

\subsection{Patient-Specific Model Parameters Estimation}

The proposed framework for detecting a personalized pulmonary trunk model in $4 \mathrm{D}$ CT and sparse cMRI data was evaluated on $504 \mathrm{D} \mathrm{CT}$ (500 volumes) and 74 sparse cMRI (74 ED Volumes associated with $4736 \mathrm{LA} / \mathrm{SA}$ planes) studies from patients with different CHD. Each volume in the data set is associated with annotation, manually generated by experts, which is considered as ground truth. Three-fold cross validation was used to divide the data set into training and test data.

Table 1 summarizes the detection performance on both modalities (CT and sparse cMRI), from the test data. Point-to-mesh measurement error was used to evaluate the detection accuracy between the ground truth and the detected model for both modalities. Average speed of 10sec per frame was achieved for both modalities on a standard 2.0GHz Dual Core PC.

Table 1. Detection accuracy

\begin{tabular}{|c|c|c|c|}
\hline CT/MRI & Mean Error $(\mathrm{mm})$ & Median $(\mathrm{mm})$ & Std.Dev $(\mathrm{mm})$ \\
\hline Landmarks & $3.5 / 4.3$ & $5.1 / 6.4$ & $2.7 / 3.0$ \\
\hline Center Line & $3.0 / 3.3$ & $2.3 / 2.3$ & $1.7 / 2.0$ \\
\hline Full Surface & $\mathbf{1 . 6 / 1 . 9}$ & $\mathbf{1 . 2} / \mathbf{1 . 3}$ & $\mathbf{0 . 2} / \mathbf{0 . 2}$ \\
\hline
\end{tabular}




\subsection{Intra-modality Comparison between CT and MRI}

The inter-modality consistency of the model was demonstrated on a subset of 10 patients which underwent both imaging investigations, 4D CT and sparse cMRI (see Fig. 2). Ground truth and detected pulmonary trunk models from both modalities were compared using the point-to-mesh measurement and clinically relevant diameter measurements: RVOT, hinges and commissures. Results are summarized in Table 2. A strong inter-modality correlation, $r=0.992, p<$ 0.0001 and confidence of $98 \%$, was obtained for CT and cMRI for the pulmonary trunk models.
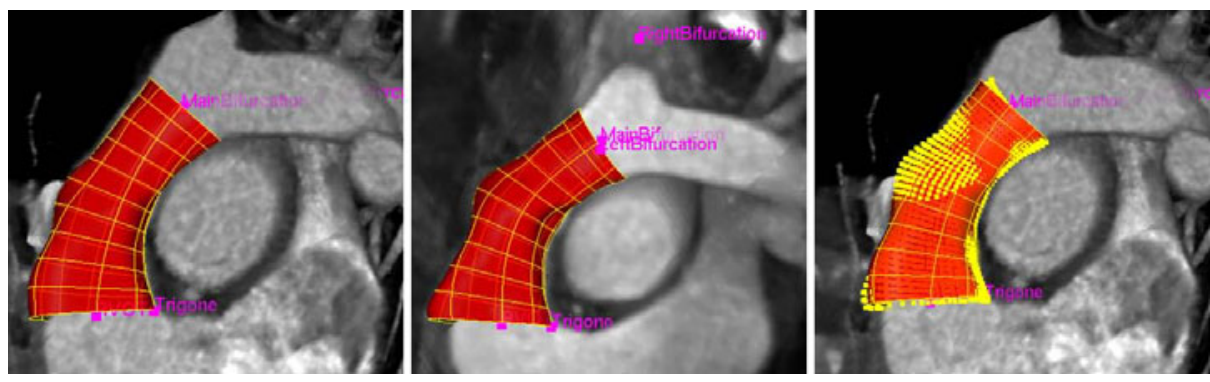

Fig. 2. Pulmonary trunk model in CT (left) and cMRI (middle) data for the ED phase. Inter-modality consistency by projecting the cMRI model(yellow points) into the CT data (right).

Table 2. Model based intra-modality comparison between CT and MRI

\begin{tabular}{|c|c|c|}
\hline$(\mathrm{mm})$ & Ground truth & Estimation \\
\hline RVOT & $0.7 \pm 0.5$ & $3.8 \pm 1.5$ \\
\hline Hinges & $1.2 \pm 1.4$ & $2.6 \pm 4.7$ \\
\hline Commissures & $1.5 \pm 1.2$ & $3.2 \pm 1.7$ \\
\hline Point-to-mesh & $1.4 \pm 0.1$ & $2.5 \pm 0.7$ \\
\hline
\end{tabular}

\subsection{Regression Based Dynamic Model Reconstruction}

As described in Sec. 1 the sparse cMRI protocol is able to capture the full anatomy of the pulmonary trunk only in the ED phase (3D volume) of the heart and parts of the pulmonary trunk in 2D planes (LA and SA) over the cardiac cycle. However, a full 4D model of the pulmonary trunk can be still computed from the available sparse data by learning the full motion from 4D CT data. For this purpose we learned a regression model as presented in Sec. 3 on a training data set of 72 4D CT (720 Volumes) studies. Two different machine-learning techniques (boosting and random forest) are used to train the regression model and to evaluate the reconstruction error. Table 3 presents reconstruction results obtained by applying the learned regression model on the sparse cMRI images and evaluating the reconstructed model on $4 \mathrm{D} \mathrm{CT}$, for a set of 10 patients, which underwent both imaging modalities. Figure 3 illustrates the reconstruction error distributed over the cardiac cycle. 
Table 3. Reconstruction error for Random Forest and Boosting

\begin{tabular}{|l|c|c|}
\hline & Boosting & Random Forest \\
\hline Mean Err. & $1.44(\mathrm{~mm})$ & $3.2(\mathrm{~mm})$ \\
\hline Std. Dev & $0.21(\mathrm{~mm})$ & $0.23(\mathrm{~mm})$ \\
\hline Speed & $3.07(\mathrm{~ms})$ & $6.21 \mathrm{~ms}$ \\
\hline
\end{tabular}

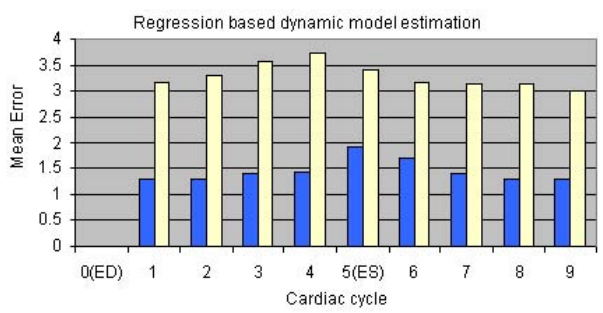

\section{Conclusion}

In this paper we propose a cross-modality detection framework for estimating a dynamic personalized model of the pulmonary trunk from the available data, $4 \mathrm{D}$ CT and sparse cMRI. A novel regression based reconstruction method is presented and used to infer the incomplete temporal information characteristic to the sparse cMRI protocols. The estimated model from both modalities can be utilized to extract morphological and functional information of the pulmonary trunk and dynamics over the cardiac cycle. Extensive experiments performed on a large heterogeneous data set demonstrated a precision of $1.44 \mathrm{~mm}$ data at a speed of 11 seconds per volume. The proposed method has the potential to significantly advance the pulmonary trunk treatment.

\section{References}

1. Bonhoeffer, P., et al.: Percutaneous insertion of the pulmonary valve. Journal of the American College of Cardiology 39(10), 1664-1669 (2002)

2. Taylor, A.: Cardiac imaging: Mr or ct? which to use when. Pediatr. Radiology 38 (2008)

3. Vitanovski, D., Ionasec, R., Georgescu, B., Huber, M., Taylor, A., Hornegger, J., Comaniciu, D.: Personalized pulmonary trunk modeling for intervention planning and valve assessment estimated from CT data. In: Yang, G.-Z., Hawkes, D., Rueckert, D., Noble, A., Taylor, C. (eds.) MICCAI 2009. LNCS, vol. 5761, pp. 17-25. Springer, Heidelberg (2009)

4. Zhou, S.K., Georgescu, B., Zhou, X.S., Comaniciu, D.: Image based regression using boosting method. In: ICCV (2005)

5. Friedman, J.H.: Greedy function approximation: A gradient boosting machine. Annals of Statistics 29, 1189-1232 (2000)

6. Breiman, L.: Random forests. In: Machine Learning, pp. 5-32 (2001)

7. Viola, P., Jones, M.: Rapid object detection using a boosted cascade of simple features. In: CVPR (2001)

8. Tu, Z.: Probabilistic boosting-tree: Learning discriminativemethods for classification, recognition, and clustering. In: ICCV (2005)

9. Zheng, Y., Barbu, A., et al.: Fast automatic heart chamber segmentation from 3d ct data using marginal space learning and steerable features. In: ICCV (2007) 